\title{
Phototransformable fluorescent proteins: future challenges
}

$\operatorname{Virgile~Adam~}^{*, 1,2,3}$, Romain Berardozzi ${ }^{1,2,3}$, Martin Byrdin ${ }^{1,2,3}$ and Dominique Bourgeois ${ }^{*}, 1,2,3$

${ }^{1}$ Université Grenoble Alpes, Institut de Biologie Structurale (IBS), F-38000 Grenoble, France ; ${ }^{2} \mathrm{CNRS}$, IBS, F-38000 Grenoble, France ; ${ }^{3} \mathrm{CEA}$, DSV, IBS, F-38000 Grenoble, France

*Authors for correspondence:

virgile.adam@ibs.fr, Tel: +33 (0)4 57428567

dominique.bourgeois@ibs.fr, Tel: +33 (0)4 57428644

\section{Abbreviations:}

dSTORM, Direct stochastic optical reconstruction microscopy; (E)GFP, (Enhanced) green fluorescent protein; FP, Fluorescent protein; LOV, Light, oxygen, or voltage-sensing flavoprotein; MEA, Monoethanolamine; PAFP, Photoactivatable fluorescent protein; PAGFP, Photoactivatable green fluorescent protein; PALM, Photoactivated Localization microscopy; PCFP, Photoconvertible fluorescent protein; Pfr, Phytochrome far-red; Pr, Phytochrome red; Pol II, RNA polymerase II; PTFP, Phototransformable fluorescent protein; RESOLFT, Reversible saturable optical fluorescence transitions; RFP, Red fluorescent protein; RNA, Ribonucleic acid; RSFP, Reversible switchable fluorescent protein; CTPALM, Time-correlated photoactivated localization microscopy; CTPA, Consecutive Two-photon absorption; UV, Ultra-violet; XFEL, X-ray free electron laser 


\section{Summary}

In fluorescence microscopy, the photophysical properties of the fluorescent markers play a fundamental role. The beauty of phototransformable fluorescent proteins (PTFPs) is that some of these properties can be precisely controlled by light. A wide range of PTFPs have been developed in recent years, including photoactivatable, photoconvertible and photoswitchable fluorescent proteins. These smart labels triggered a plethora of advanced fluorescence methods to scrutinize biological cells or organisms dynamically, quantitatively and with unprecedented resolution. Despite continuous improvements, PTFPs still suffer from limitations, and mechanistic questions remain as to how these proteins precisely work.

\section{Introduction}

Fluorescent proteins are central to biology, allowing specific, multicolor and nondisruptive labeling in living cells, tissues and whole organisms $[1,2]$. Yet, they display highly complex photophysics. For example, it was shown that GFP itself can undergo photoactivation [3], photoconversion [4] and photoswitching [5], in addition to blinking [6], and photobleaching [7]. These low-yield processes have mostly been perceived as nuisances, until the discovery of fluorescent proteins from Anthozoa species completely changed our view. Starting with Kaede in Trachyphyllia geoffroyi and EosFP in Lobophyllia hemprichii, well-behaved green-to-red photoconvertible FPs soon suggested a huge potential for dynamical tracking experiments and super-resolution localization microscopy, and were rapidly developed into a whole palette of highly performing variants. Likewise, after the discovery of the reversibly switchable asFP595 in Anemonia sulcata, the engineering of monomeric Dronpa from Echinophyllia sp. revolutionized the field, and was followed by the release of many variants opening up a continuously growing panel of applications, from biotechnology to optogenetics. In the meantime, GFP itself was turned into a highly efficient photoactivatable protein named PA-GFP and different hues followed, adequate for e.g. pulse-chase microscopy approaches. PTFPs developed in the last two years are summarized in Table 1. The present paper mainly focuses on future challenges in the field of PTFPs. For a comprehensive view on PTFP's mechanisms, engineering and applications, the reader is directed to the many exhaustive reviews published recently $[1,2,8-12]$. We start by 
recapitulating the three different classes of PTFPs, emphasizing recent mechanistic insight. Then, based on three fascinating examples from recent literature, we highlight important flaws that PTFPs still face today. We list a series of mechanistic questions still to be investigated, and we propose perspectives for further developments of PTFPs.

\section{PTFPs classes and mechanisms}

In PTFPs, three main classes are distinguished. Photoactivatable fluorescent proteins (PAFPs) undergo non-reversible light-induced activation from a non-fluorescent to a fluorescent state; photoconvertible FPs (PCFPs) exhibit non-reversible conversion between two fluorescent states with distinct emission colors; and reversibly switchable FPs (RSFPs) can be photoswitched back and forth between a fluorescent on-state and a non-fluorescent off-state. In PAFPs and PCFPs, the phototransformations involve covalent modifications of the FP whereas in RSFPs only conformational rearrangements of the chromophore and its environment take place. The basic mechanisms involved are illustrated in figure 1. It should be noted that PCFPs and RSFPs of anthozoan origin often share remarkably high sequence identity and structural similarity. This allowed the development of PTFPs combining both photoconversion and photoswitching properties [13] and the re-engineering of PCFPs into RSFPs [14] or vice versa [15].

\section{PAFPs: photoactivatable fluorescent proteins}

PATagRFP [16] and PAmKate [17] are the latest introduced PAFPs, with a red (595 nm) and far red $(628 \mathrm{~nm})$ fluorescence emission in their activated state, respectively. Despite a relatively low brightness, these two proteins could successfully be used in multicolor localization microscopy experiments $[16,17]$. Whereas the photoactivation mechanisms of PA-GFP and PAmCherry are relatively well-established, involving decarboxylation of the fully conserved Glu222 through photo-Kolbe reactions, those of PATagRFP and PAmKate remain to be deciphered. In PATagRFP, the presence of oxygen is required to promote chromophore oxidation, like in PAmCherry. The reaction involves uncharacterized intermediate states and the consecutive absorption of twonear-UV photons (CTPA, e.g. $405 \mathrm{~nm}$ ) [16], not to be confused with the simultaneous absorption of two photons, commonly referred as TPA. In 
fact, Glu222 decarboxylation in PA-GFP is also mainly a CTPA process [18,19], raising the question how weak 405-nm light still activates PA-GFP. Interestingly, Glu222 decarboxylation can cause photobleaching in some anthozoan PCFPs or RSFPs [20], instead of photoactivation, which suggests the existence of two parallel photoactivation mechanisms.

\section{PCFPs: photoconvertible fluorescent proteins}

Whereas well-known green-to-red PCFPs emit below $600 \mathrm{~nm}$ in their photoconverted state, the recently introduced PSmOrange [21] and PSmOrange2 [22] shift their emissions from orange to the dark red region $(660 \mathrm{~nm})$ upon illumination at $488 \mathrm{~nm}$. The photoconversion mechanisms of these two proteins remain to be precisely established but appear to involve CTPA, like PATagRFP, as well as the presence of oxidizing agents in the nanoenvironment. This is in stark contrast to the mechanism of Kaede- or Eos-like PCFPs, in which backbone breakage and elongation of the chromophoric $\pi$-system results from absorption of a single UV-violet photon and can be prevented by addition of electron acceptors in the medium [23]. The engineering of a least-evolved ancestral PCFP [24] and ultrafast spectroscopic investigations $[25,26]$ contributed to clarify the mechanism by which green-to-red PCFPs photoconvert. Evidence is growing that a histidine-containing and protonated chromophore embedded in a "typical" anthozoan-FP chromophore pocket does not guarantee efficient photoconversion and that the local charge network [24] as well as global conformational dynamics e.g. due to the oligomeric structure of PCFPs [15] play key roles. In Dendra2, the mechanism by which the anionic chromophore may also promote photoconversion remains unclear. Of major concern is the observation that photoconversion in green-to-red PCFPs is always incomplete (meaning that only a fraction of PCFPs in a sample is able to achieve photoconversion) [27], complicating quantitative molecular counting using PALM (see [9] and Figure $\mathbf{2 b}$ ). The mechanistic reason, and a possible cure, for such incomplete photoconversion are still lacking.

\section{RSFPs: reversibly switchable (or photochromic) fluorescent proteins}

RSFPs offer the widest range of applications [10,12], and therefore many efforts have been devoted recently to extend their panel [13-15,22,28-31] and tackle their mechanisms 
[15,20,32-35]. Photoswitching in RSFPs is generally based on an intrinsic property of theisolated chromophore: light-induced cis-trans isomerization. Therefore photoswitching tends to naturally occur to some extent in many FPs, and has been successfully expanded by engineering in both hydrozoan and anthozoan RSFPs. Depending on whether photon absorption at the peak excitation wavelength induces off-switching, on-switching, or has no switching effect, RSFPs are referred to as negative [36], positive [37], or decoupled [38], respectively, each having their pros and cons. Recent findings concern the heavily debated mechanism by which chromophore isomerization couples to chromophore protonation in negative or positive anthozoan switchers. Several pieces of evidence from ultrafast IR and UV-vis spectroscopy now suggest that (de)protonation of the chromophore in Dronpa [35] or Padron [33] is a ground state process that follows isomerization, although other authors disagree [34]. Another interesting finding is that negative switchers such as Padron are capable of efficient photoswitching at cryogenic temperatures [32], potentially opening up nanoscopy applications at such low temperatures. Recent engineering efforts have also aimed at increasing the switching speed and fatigue resistance (i.e. the number of achievable photo cycles before photobleaching) of RSFPs of use for e.g. RESOLFT applications [28,39] (see below and Figure 2c). Blue and infrared RSFPs have been developed that, despite their low brightness, offer exciting perspectives for extracting weak signals by demodulation [29] or difference imaging (see [40] and Figure 2a) in conditions of high autofluorescence.

\section{PTFPs applications}

PTFPs are presently used in a very wide panel of applications [1,2,8-12] that cannot be exhaustively reviewed here. One recent evolution is that attention is now often paid to optimizing PTFPs for several applications instead of only one, allowing to obtain complementary results with a single construct $[15,41,42]$. In this regard, bi-photochromic PTFPs such as NijiFP or Dendra2-M159A [13] or three-way highlighters like pcDronpa2 [15] are versatile members that may offer novel applications in the future.

In the following, we take advantage of three outstanding papers to highlight three major challenges that PTFPs' research will face in the next years. 


\section{Expanding the PTFPs toolbox with infrared-emitting members}

Far-red-shifted variants are a necessary ingredient in deep-tissue or live-animal imaging. In a recent tour-de-force, Piatkevitch et al. [40] developed an infrared-RSFP by exploiting the photocycle of phytochromes between red $(\mathrm{Pr})$ and far-red (Pfr) states. The photosensory core domain of a bacterial phytochrome was engineered to provide PAiRFP1 and PAiRFP2, which can be reversibly switched by 660-nm light into a Pfr-like state emitting fluorescence at $718 \mathrm{~nm}$. Despite their low fluorescence quantum yield and suboptimal contrast ratio (fluorescence in the off state amounts to $20 \%$ of that in the on-state), the two proteins facilitate deep-tissue imaging: the difference between images recorded in the on and off state of the markers can be computed to highlight e.g. a labeled tumor region (Figure 2a). In general, far-red and infrared FPs all suffer from a low fluorescence quantum yield ( $\sim 5 \%$ for PAiRFP1/2), possibly due to the many degrees of freedom in elongated chromophores, promoting a multitude of non-radiative de-excitation pathways. Thus far, iPTFPs are therefore inappropriate for single molecule detection and a challenge for the future will be to engineer iPTFPs of high-brightness suitable for localization microscopy.

\section{Mastering Dendra2 photophysics to monitor transient clustering of RNA polymerase in live} cells

Localization microscopy has recently turned into a quantitative method to count macromolecules within cells, assess their stoichiometry or monitor their clustering pattern. The work by Cisse et al [43] illustrates this trend, introducing time-correlated PALM (CTPALM) to dynamically measure the clustering of RNA polymerase II (Pol II) in the nuclei of live human cells. By analyzing the temporal sequence of single-molecule detections in individual clusters, it was shown that "nuclear transcription factories" made of Pol II clusters continuously assemble and disassemble (Figure $\mathbf{2 b}$ ). To confidently interpret the results, Cisse et al, however, had to pay critical attention to the complex photophysics of the employed PCFP marker, Dendra2. Like many other PCFPs, Dendra2 stochastically switches to long-lived dark states once photoconverted, which confuses quantitative studies because each molecule may be counted more than once. A number of methods have been proposed to tackle this difficulty, but they are all statistical by nature and thus remain imperfect [9]. 
There is yet no clear understanding of the exact mechanism giving rise to this slow blinking, and the nano-environmental sensitivity of the process remains to be assessed. A combination of structural $[44,45]$ and single-molecule $[46,47]$ data on mEos2, Dendra2 and IrisFP suggests that a blinking mechanism in anthozoan PTFPs could involve a transient protonation of carbon $C_{\alpha}$ of the chromophore methylene bridge via the nearby Arg66 (Figure 3a). Alternatively, the long dark states could relate to a residual propensity to cistrans chromophore isomerization. Another difficulty in Cisse et al is that the exact numbers of Pol II molecules forming the clusters could not be extracted because of the suboptimal photoconversion efficiency of Dendra2: a number of labeled Pol II molecules never get photoconverted and are thus not counted. Elegant methods have been proposed recently to evaluate the problem in various PCFPs [27] and to correct for it [48], but the underlying photophysics remains mysterious.

Thus, challenges for the future will be to better understand the complex blinking and photoconversion behavior of PCFPs and engineer low-blinking and fully photoconvertible variants.

\section{Video-rate super-resolution microscopy with RSFPs}

The concomitant development of sophisticated optical setups and dedicated RSFPs has considerably advanced the nanoscopy approach named RESOLFT $[28,39,49]$. By employing RSFPs with long on- and off-state lifetimes, RESOLFT requires considerably less light than STED nanoscopy to break the diffraction barrier, but at the expense of a reduced timeresolution. However, employing RSFPs with high switching speed such as Dronpa-M159T [39] or rsEGFP2 [28] allowed relatively fast dynamic RESOLFT imaging. Chmyrov et al [49] demonstrated that video-rate RESOLFT nanoscopy could be achieved on very large field-ofviews by designing a highly parallelized setup based on orthogonal and incoherently crossed standing light waves. The spectacular images (Figure 2c) were obtained with rsEGFP(N205S), a slow switching variant of rSEGFP that, by emitting about twice the number of photons per cycle, allowed obtaining images of excellent signal-to-noise ratio. Slow-switching variants such as rsEGFP(N205S) deliver high quality images but limit the achievable acquisition rate, which in the present case was more than compensated by the parallelized optical setup. Noteworthy, the light intensity and the cellular environment influence the switching 
properties of RSFPs [28] and many other photophysical parameters (e.g. brightness, expression level, maturation rate, contrast ratio) determine the achievable image quality. Another critical aspect in RESOLFT is that the resolution enhancement is limited by the fatigue resistance of the RSFP, calling for more photostable RSFPs (and PTFPs in general). Progress in this direction has been achieved by directed evolution [28] and by recent investigations on IrisFP revealing that that two completely different photobleaching mechanisms occur in anthozoan PTFPs [20]. At light levels typical of standard widefield microscopy $\left(\ll 100 \mathrm{~W} / \mathrm{cm}^{2}\right)$, IrisFP photobleaching involves an oxygen-dependent mechanism, whereas at light levels employed in PALM microscopy $\left(>100 \mathrm{~W} / \mathrm{cm}^{2}\right)$ an oxygen-independent CTPA process dominates (Figure 3b,c). It remains unknown whether similar scenarios occur in hydrozoan PTFPs such as rsEGFP or at higher light levels typical of RESOLFT nanoscopy.

Overall, challenges for the future will be to rationally design high-contrast-ratio, fastswitching, high-brightness and fatigue-resistant RSFPs.

\section{Further mechanistic questions}

Fluorescent molecules are highly sensitive to their environment. While the role of the environment surrounding organic dyes has drawn considerable research efforts, for example to develop dSTORM nanoscopy [50], its importance in PTFPs has been underestimated. Yet, the $\beta$-barrel embedding the chromophore in FPs is generally flexible and permeable. Oxygen $[16,21,51]$ and small buffer components such as ethylene glycol [52] can reach the chromophore, while vitamins in the medium may accelerate photobleaching [53]. There is more and more evidence that the efficiency of phototransformations are influenced by the cellular and/or redox microenvironment, as shown for the oxidative redding of EGFP $[23,53]$, the photoconversion of mEos2 [54], or the photoswitching of rsEGFP variants [28]. However, the precise proton and electron pathways, oxygen routes or role of cavities within PTFP structures remain to be determined. Influences of the solvent on the blinking and bleaching behaviors of PTFPs have also been observed, e.g. presence of redox active molecules such as MEA [55]. In all these cases, the barrel could act as relaying networks via $\mathrm{H}$-bonds or redox active amino acids. Further deciphering such mechanisms PTFPs will necessitate the development of new theoretical and experimental approaches. Quantitative in cellulo and in 
vitro investigations of PTFP's photophysics at the single-molecule and ensemble levels should be pursued jointly, possibly in combination with nanofluidic approaches or optimized sol-gel matrices to cleanly control the PTFP nanoenvironment. Modeling methods would have to be adapted to adequately reproduce low yield processes while taking into account environmental factors. Amazing new perspectives for ultrafast structural investigations seem to be ahead by using X-ray free-electron lasers (XFEL) for visible pump/X-ray probe experiments on PTFPs.

\section{Perspectives for future PTFPs}

Fluorescence appears much more widespread in oceans than previously thought, as even vertebrates such as fishes have been found to emit fluorescence [56]. Putative FPs of these organisms probably undergo entirely different fluorescence mechanisms than those involved in GFP-like types, thus offering the opportunity for considerable enlargement of the FP toolbox. A recent example is UnaG, a protein of about half the size of GFP, discovered in a freshwater eel and that fluoresces green upon reversible binding of bilirubin [57]. Another alternative to conventional FPs resides in the increasing use of Light, Oxygen, or Voltagesensing (LOV) flavoprotein domains [58] whose covalently linked flavin chromophore does not require oxygen to maturate. Amongst other advantages, their small size allows the exploration of tight cellular compartments as recently demonstrated for bacterial periplasm [59]. Engineering these new types of FPs so that they become phototransformable represents a future quest.

Yet, existing PTFPs have still much to offer and will continue to be refined by genetic engineering. Underappreciated improvements such as codon optimizations or adaptations of $\mathrm{C}$ and $\mathrm{N}$ termini are possible solution to rescue non-functional genetic fusions and to explore "FP-hostile" (e.g. acidic or oxidative) organelles or organisms.

PTFPs also need to see their color palette expanded. The incorporation of non-natural amino acids is a promising route as they can form novel chromophores [60,61]. Plotting the brightness of PTFPs versus their emission color (Figure 4), it appears that, despite recent efforts $[29,40]$, most PTFPs concentrate nowadays in the green and orange range of emission, leaving the violet/blue and red/far red regions weakly populated. Even more 
strikingly, the few PTFPs engineered from yellow FPs such as EYQ1 [62] or Dreiklang [38] ended up with green variants and it appears that, unlike conventional FPs, yellowish PTFPs emitting between 530 and $560 \mathrm{~nm}$ are totally missing. This absence is particularly noteworthy since this is precisely the wavelength range where the brightness is expected to be the highest [1] so that the engineering of bright yellow PTFPs remains a challenge.

In conclusion, unfolding the full potential of PTFPs will continue to keep developers busy for a while -provided continued funding is made available- !

\section{Acknowledgments}

The authors acknowledge V. Verkhusha, I. Izeddin and A. Chmyrov for providing raw images to adapt figure 2. Institutional grants from the CNRS, CEA and UJF and financial support by the ANR (ANR-2011-BSV5-012-01 NOBLEACH) are acknowledged.

\section{References and recommended reading}

Papers of particular interest, published within the period of review, have been highlighted as:

- of special interest

$\bullet \bullet$ of outstanding interest

1. Dedecker P, De Schryver FC, Hofkens J: Fluorescent proteins: shine on, you crazy diamond. J Am Chem Soc 2013, 135:2387-2402.

2. Nienhaus K, Ulrich Nienhaus G: Fluorescent proteins for live-cell imaging with superresolution. Chem Soc Rev 2014, 43:1088-1106.

3. van Thor JJ, Gensch T, Hellingwerf KJ, Johnson LN: Phototransformation of green fluorescent protein with $\mathrm{UV}$ and visible light leads to decarboxylation of glutamate 222. Nat Struct Biol 2002, 9:37-41.

4. Elowitz MB, Surette MG, Wolf PE, Stock J, Leibler S: Photoactivation turns green fluorescent protein red. Curr Biol 1997, 7:809-812.

5. Sinnecker D, Voigt P, Hellwig N, Schaefer M: Reversible photobleaching of enhanced green fluorescent proteins. Biochemistry 2005, 44:7085-7094.

6. Dickson RM, Cubitt AB, Tsien RY, Moerner WE: On/off blinking and switching behaviour of single molecules of green fluorescent protein. Nature 1997, 388:355358.

7. White J, Stelzer E: Photobleaching GFP reveals protein dynamics inside live cells. Trends Cell Biol 1999, 9:61-65. 
8. Zhou XX, Lin MZ: Photoswitchable fluorescent proteins: ten years of colorful chemistry and exciting applications. Curr Opin Chem Biol 2013, 17:682-690.

9. Sengupta P, van Engelenburg SB, Lippincott-Schwartz J: Superresolution imaging of biological systems using photoactivated localization microscopy. Chem Rev 2014, 114:3189-3202.

10. Bourgeois $\mathrm{D}$, Adam $\mathrm{V}$ : Reversible photoswitching in fluorescent proteins: a mechanistic view. IUBMB Life 2012, 64:482-491.

11. Bourgeois D, Regis-Faro A, Adam V: Photoactivated structural dynamics of fluorescent proteins. Biochem Soc Trans 2012, 40:531-538.

12. Adam V: Phototransformable fluorescent proteins: which one for which application? Histochem Cell Biol 2014.

13. Adam V, Moeyaert B, David CC, Mizuno H, Lelimousin M, Dedecker P, Ando R, Miyawaki A, Michiels J, Engelborghs Y, et al.: Rational design of photoconvertible and biphotochromic fluorescent proteins for advanced microscopy applications. Chem Biol 2011, 18:1241-1251.

14. Chang H, Zhang M, Ji W, Chen J, Zhang Y, Liu B, Lu J, Zhang J, Xu P, Xu T: A unique series of reversibly switchable fluorescent proteins with beneficial properties for various applications. Proc Natl Acad Sci U S A 2012, 109:4455-4460.

- 15. Moeyaert B, Nguyen Bich N, De Zitter E, Rocha S, Clays K, Mizuno H, van Meervelt L, Hofkens J, Dedecker P: Green-to-red photoconvertible Dronpa mutant for multimodal super-resolution fluorescence microscopy. ACS Nano 2014, 8:16641673.

Directed-evolution and structure-driven rational design were employed to design pcDronpa2, a highbrightness (unfortunately tetrameric) three-way highlighter that combines green-to-red photoconversion and photochromism in the green state. A large panel of methods, from crystallography to single-molecule localization microscopy were used and interesting mechanistic considerations are proposed.

16. Subach FV, Patterson GH, Renz M, Lippincott-Schwartz J, Verkhusha VV: Bright monomeric photoactivatable red fluorescent protein for two-color superresolution sptPALM of live cells. J Am Chem Soc 2010, 132:6481-6491.

17. Gunewardene MS, Subach FV, Gould TJ, Penoncello GP, Gudheti MV, Verkhusha VV, Hess ST: Superresolution imaging of multiple fluorescent proteins with highly overlapping emission spectra in living cells. Biophys $J$ 2011, 101:1522-1528.

18. Langhojer F, Dimler F, Jung G, Brixner T: Ultrafast photoconversion of the green fluorescent protein studied by accumulative femtosecond spectroscopy. Biophys $J$ 2009, 96:2763-2770.

19. Ding L, Chung LW, Morokuma K: Reaction mechanism of photoinduced decarboxylation of the photoactivatable green fluorescent protein: an ONIOM(QM:MM) study. J Phys Chem B 2013, 117:1075-1084.

- 20 . Duan C, Adam V, Byrdin M, Ridard J, Kieffer-Jaquinod S, Morlot C, Arcizet D, Demachy I, Bourgeois D: Structural evidence for a two-regime photobleaching mechanism in a reversibly switchable fluorescent protein. J Am Chem Soc 2013, 135: $15841-15850$. 
the type of damage inflicted on a PTFP by visible light is dependent on its intensity: at low flux an oxygen-dependent mechanism prevails, whereas higher flux favors reductive chromophore destruction.

21. Subach OM, Patterson GH, Ting LM, Wang Y, Condeelis JS, Verkhusha VV: A photoswitchable orange-to-far-red fluorescent protein, PSmOrange. Nat Methods 2011, 8:771-777.

- 22. Subach OM, Entenberg D, Condeelis JS, Verkhusha VV: A FRET-facilitated photoswitching using an orange fluorescent protein with the fast photoconversion kinetics. J Am Chem Soc 2012, 134:14789-14799.

Optimizing absorption wavelength and photoconversion rate of the orange-to-deep-red switcher $P S m O r a n g e$, the authors succeed in triggering the photoconversion by FRET from a green emitting $F P$, thus creating a "memory" of a transient interaction.

23. Saha R, Verma PK, Rakshit S, Saha S, Mayor S, Pal SK: Light driven ultrafast electron transfer in oxidative redding of green fluorescent proteins. Sci Rep 2013, 3:1580.

- 24 . Kim H, Grunkemeyer TJ, Modi C, Chen L, Fromme R, Matz MV, Wachter RM: Acid-base catalysis and crystal structures of a least evolved ancestral GFP-like protein undergoing green-to-red photoconversion. Biochemistry 2013, 52:80488059.

13 mutations were shown to be necessary and sufficient to evolve GFP into a hydrozoan PCFP. Structural and biochemical studies convincingly suggest a green-to-red photoconversion mechanism in which Glu222 abstracts a proton from His65 in the excited state and transfers it to Phe64 via Gln42 to promote bond cleavage and chromophore extension. The quadrupolar network consisting of Glu222, His203, Glu148 and Arg69, as well as the residue Gln42 are proposed to play an essential role in the process.

25. Fron E, Van der Auweraer M, Moeyaert B, Michiels J, Mizuno H, Hofkens J, Adam V: Revealing the excited-state dynamics of the fluorescent protein Dendra2. $J$ Phys Chem B 2013, 117:2300-2313.

26. Fron E, Sliwa M, Adam V, Michiels J, Rocha S, Dedecker P, Hofkens J, Mizuno H: Excited state dynamics of the photoconvertible fluorescent protein Kaede revealed by ultrafast spectroscopy. Photochem Photobiol Sci 2013.

- 27. Durisic N, Laparra-Cuervo L, Sandoval-Alvarez A, Borbely JS, Lakadamyali M: Single-molecule evaluation of fluorescent protein photoactivation efficiency using an in vivo nanotemplate. Nat Methods 2014, 11:156-162.

An interesting example of in cellulo evaluation of PTFP's photophysics at the single molecule level. A nanotemplate approach allowed to precisely evaluate the photoconversion and photoactivation efficiencies of a variety of PCFPs and PAFPs. A maximum of $60 \%$ photoconversion efficiency was demonstrated for mEos2.

- 28. Grotjohann T, Testa I, Reuss M, Brakemann T, Eggeling C, Hell SW, Jakobs S: rsEGFP2 enables fast RESOLFT nanoscopy of living cells. eLife 2012, 1:e00248.

In a mutagenesis effort, the authors obtained a double mutant of their EGFP-based reversibly switchable fluorescent protein (rsEGFP) whose photoswitching efficiency is improved. The resulting variant called rsEGFP2 makes it possible to accelerate the RESOLFT acquisition times up to 250 times. Furthermore, with a similar brightness as compared to its parent, rsEGFP2 is two times more 
resistant to photofatigue and its chromophore is 9 times faster to achieve maturation.

29. Jablonski AE, Vegh RB, Hsiang JC, Bommarius B, Chen YC, Solntsev KM, Bommarius AS, Tolbert LM, Dickson RM: Optically modulatable blue fluorescent proteins. $J$ Am Chem Soc 2013, 135:16410-16417.

30. Lavoie-Cardinal F, Jensen NA, Westphal V, Stiel AC, Chmyrov A, Bierwagen J, Testa I, Jakobs S, Hell SW: Two-color RESOLFT nanoscopy with green and red fluorescent photochromic proteins. Chemphyschem 2014, 15:655-663.

31. Zhang M, Chang H, Zhang Y, Yu J, Wu L, Ji W, Chen J, Liu B, Lu J, Liu Y, et al.: Rational design of true monomeric and bright photoactivatable fluorescent proteins. Nat Methods 2012, 9:727-729.

32. Faro AR, Carpentier P, Jonasson G, Pompidor G, Arcizet D, Demachy I, Bourgeois D: Low-temperature chromophore isomerization reveals the photoswitching mechanism of the fluorescent protein Padron. J Am Chem Soc 2011, 133:1636216365.

33. Fron E, Van der Auweraer M, Hofkens J, Dedecker P: Excited state dynamics of photoswitchable fluorescent protein Padron. J Phys Chem B 2013, 117:1642216427.

34. Gayda S, Nienhaus $\mathrm{K}$, Nienhaus GU: Mechanistic insights into reversible photoactivation in proteins of the GFP family. Biophys $J$ 2012, 103:2521-2531.

35. Warren MM, Kaucikas M, Fitzpatrick A, Champion P, Sage JT, van Thor JJ: Groundstate proton transfer in the photoswitching reactions of the fluorescent protein Dronpa. Nat Commun 2013, 4:1461.

36. Ando R, Mizuno H, Miyawaki A: Regulated fast nucleocytoplasmic shuttling observed by reversible protein highlighting. Science 2004, 306:1370-1373.

37. Andresen M, Stiel AC, Folling J, Wenzel D, Schonle A, Egner A, Eggeling C, Hell SW, Jakobs S: Photoswitchable fluorescent proteins enable monochromatic multilabel imaging and dual color fluorescence nanoscopy. Nat Biotechnol 2008, 26:10351040 .

38. Brakemann T, Stiel AC, Weber G, Andresen M, Testa I, Grotjohann T, Leutenegger M, Plessmann U, Urlaub H, Eggeling C, et al.: A reversibly photoswitchable GFP-like protein with fluorescence excitation decoupled from switching. Nat Biotechnol 2011, 29:942-947.

39. Testa I, Urban NT, Jakobs S, Eggeling C, Willig KI, Hell SW: Nanoscopy of living brain slices with low light levels. Neuron 2012, 75:992-1000.

40. Piatkevich KD, Subach FV, Verkhusha VV: Far-red light photoactivatable nearinfrared fluorescent proteins engineered from a bacterial phytochrome. Nat Commun 2013, 4:2153.

41. McEvoy AL, Hoi H, Bates M, Platonova E, Cranfill PJ, Baird MA, Davidson MW, Ewers H, Liphardt J, Campbell RE: mMaple: a photoconvertible fluorescent protein for use in multiple imaging modalities. PLoS One 2012, 7:e51314.

42. Jensen NA, Danzl JG, Willig KI, Lavoie-Cardinal F, Brakemann T, Hell SW, Jakobs S: Coordinate-targeted and coordinate-stochastic super-resolution microscopy with 
the reversibly switchable fluorescent protein Dreiklang. Chemphyschem 2014, 15:756-762.

43. Cisse II, Izeddin I, Causse SZ, Boudarene L, Senecal A, Muresan L, Dugast-Darzacq C, Hajj B, Dahan M, Darzacq X: Real-time dynamics of RNA polymerase II clustering in live human cells. Science 2013, 341:664-667.

44. Adam V, Lelimousin M, Boehme S, Desfonds G, Nienhaus K, Field MJ, Wiedenmann J, McSweeney S, Nienhaus GU, Bourgeois D: Structural characterization of IrisFP, an optical highlighter undergoing multiple photo-induced transformations. Proc Natl Acad Sci U S A 2008, 105:18343-18348.

45. Roy A, Field MJ, Adam V, Bourgeois D: The nature of transient dark states in a photoactivatable fluorescent protein. J Am Chem Soc 2011, 133:18586-18589.

46. Lee SH, Shin JY, Lee A, Bustamante C: Counting single photoactivatable fluorescent molecules by photoactivated localization microscopy (PALM). Proc Natl Acad Sci U S A 2012, 109:17436-17441.

47. McKinney SA, Murphy CS, Hazelwood KL, Davidson MW, Looger LL: A bright and photostable photoconvertible fluorescent protein. Nat Methods 2009, 6:131-133.

48. Puchner EM, Walter JM, Kasper R, Huang B, Lim WA: Counting molecules in single organelles with superresolution microscopy allows tracking of the endosome maturation trajectory. Proc Natl Acad Sci U S A 2013, 110:16015-16020.

49. Chmyrov A, Keller J, Grotjohann T, Ratz M, d'Este E, Jakobs S, Eggeling C, Hell SW: Nanoscopy with more than 100,000 'doughnuts'. Nat Methods 2013, 10:737-740.

50. van de Linde S, Sauer M: How to switch a fluorophore: from undesired blinking to controlled photoswitching. Chem Soc Rev 2014, 43:1076-1087.

51. Regmi CK, Bhandari YR, Gerstman BS, Chapagain PP: Exploring the diffusion of molecular oxygen in the red fluorescent protein mCherry using explicit oxygen molecular dynamics simulations. J Phys Chem B 2013, 117:2247-2253.

52. von Stetten D, Batot GO, Noirclerc-Savoye M, Royant A: Alteration of fluorescent protein spectroscopic properties upon cryoprotection. Acta Crystallogr D Biol Crystallogr 2012, 68:1578-1583.

53. Bogdanov AM, Kudryavtseva EI, Lukyanov KA: Anti-fading media for live cell GFP imaging. PLoS One 2012, 7:e53004.

54. Izeddin I, Specht CG, Lelek M, Darzacq X, Triller A, Zimmer C, Dahan M: Superresolution dynamic imaging of dendritic spines using a low-affinity photoconvertible actin probe. PLoS One 2011, 6:e15611.

55. Endesfelder U, Malkusch S, Flottmann B, Mondry J, Liguzinski P, Verveer PJ, Heilemann $\mathrm{M}$ : Chemically induced photoswitching of fluorescent probes--a general concept for super-resolution microscopy. Molecules 2011, 16:3106-3118.

56. Sparks JS, Schelly RC, Smith WL, Davis MP, Tchernov D, Pieribone VA, Gruber DF: The covert world of fish biofluorescence: a phylogenetically widespread and phenotypically variable phenomenon. PLoS One 2014, 9:e83259.

57. Kumagai A, Ando R, Miyatake H, Greimel P, Kobayashi T, Hirabayashi Y, Shimogori T, Miyawaki A: A bilirubin-inducible fluorescent protein from eel muscle. Cell 2013, 153:1602-1611. 
58. Wingen M, Potzkei J, Endres S, Casini G, Rupprecht C, Fahlke C, Krauss U, Jaeger KE, Drepper T, Gensch T: The photophysics of LOV-based fluorescent proteins - new tools for cell biology. Photochem Photobiol Sci 2014.

59. Zhang LC, Risoul V, Latifi A, Christie JM, Zhang CC: Exploring the size limit of protein diffusion through the periplasm in cyanobacterium Anabaena sp. PCC 7120 using the 13 kDa iLOV fluorescent protein. Res Microbiol 2013, 164:710-717.

60. Liu X, Li J, Hu C, Zhou Q, Zhang W, Hu M, Zhou J, Wang J: Significant expansion of the fluorescent protein chromophore through the genetic incorporation of a metal-chelating unnatural amino acid. Angew Chem Int Ed Engl 2013, 52:48054809 .

61. Groff D, Wang F, Jockusch S, Turro NJ, Schultz PG: A new strategy to photoactivate green fluorescent protein. Angew Chem Int Ed Engl 2010, 49:7677-7679.

62. Abbruzzetti S, Bizzarri R, Luin S, Nifosi R, Storti B, Viappiani C, Beltram F: Photoswitching of E222Q GFP mutants: "concerted" mechanism of chromophore isomerization and protonation. Photochem Photobiol Sci 2010, 9:1307-1319. 


\section{Figure captions:}

Fig. 1: Basic mechanisms for the three classes of PTFPs. (a) PAFPs: the case of PA-GFP is shown where the Glu222 is decarboxylated and the chromophore is activated to an anionic/fluorescent form ( $R=$ serine). (b) PCFPs: the case of EosFP is shown, where photoinduced cleavage of the backbone extends the conjugated system (R=histidine). (c) RSFPs: the case of Dronpa is shown where the chromophore can be photoswitched between a cis anionic fluorescent form and a trans protonated non-fluorescent form ( $R=$ cysteine). Important features are depicted in red.

Fig. 2: Using PTFPs for deep-tissue imaging, counting macromolecules in dense clusters and developing video-rate nanoscopy.

(a) In vivo imaging using PAiRFP1. Overlay of bright-field and fluorescence images of a control mouse (left) and mice bearing a 10-day old PAiRFP1-expressing tumor before and after photoactivation. The difference between 'non-photoactivated' and 'photoactivated' images emphasizes the contrast enhancement allowed by PAiRFP1. Reproduced from [40], with permission. (b) PALM image of Dendra2-RPB1 depicting the heterogeneous distribution of Pol II in the nucleus, with zooms on two Pol II clusters. The time-dependent detection of Dendra2 molecules in a Pol II cluster and the corresponding cumulative detection profile are shown. Reproduced from [43] with permission and with data kindly provided by I. Izeddin. (c) Live-cell imaging with parallelized RESOLFT nanoscopy using the N205S-variant of rsEGFP in a living PtK2 cell expressing the keratin19-rsEGFP(N205S) fusion protein. A zoom and a profile showing the high signal-to-noise ratio achieved are shown. Reproduced from [49], with permission and with data kindly provided by A. Chmyrov.

Fig. 3: Phototransformation mechanisms associated with blinking and bleaching in IrisFP. (a) Blinking involves a thermally-reversible photoinduced proton exchange between Arg66 and the methylene bridge of the chromophore. (b) Bleaching under weak-illumination involves an oxygen-dependent mechanism that results in sulfoxidation of the Met159 and trapping of the chromophore in a protonated/non-fluorescent form. (c) Bleaching under strong-illumination conditions results in the decarboxylation of Glu212, conformational 
change of the chromophore pocket and disordering of the chromophore in a $s p^{3}$ hybridization state ( $R=$ histidine). Important features are depicted in red.

Fig. 4: Brightness distribution of conventional FPs and PTFPs as a function of their emission colors. (a) Conventional FPs and (b) PTFPs are represented by points and labels that are colored to the approximate appearance to the naked eye. The dashed bell-shaped curves highlight the general distributions of brightness. This figure is an extended version of a figure inspired by [1]. Note that a few labels are omitted for sake of readability and that PTFPs are represented in their activated form only. The absence of PTFPs emitting in the 530-560-nm window is apparent. The histograms (insets) represent the distribution of FPs and PTFPs as a function of their emission wavelengths (bin-width $=15 \mathrm{~nm}$ ). 
Table 1 - Properties of important PTFPs developed during the last two years.

\begin{tabular}{|c|c|c|c|c|c|c|c|c|c|c|c|c|c|c|c|c|}
\hline $\begin{array}{c}\text { Protein } \\
\text { (oligomerization) }\end{array}$ & $\begin{array}{c}\text { Parent } \\
\text { protein } \\
\text { [wild-type] }\end{array}$ & $\begin{array}{c}\text { Source } \\
\text { organism }\end{array}$ & $\begin{array}{c}\text { Chromo- } \\
\text { phore }\end{array}$ & \multicolumn{2}{|c|}{$\lambda_{\max } \operatorname{ex} / \mathbf{e m}(\mathrm{nm})^{\mathrm{a}}$} & \multicolumn{2}{|c|}{$\varepsilon\left(\mathrm{mM}^{-1} \cdot \mathrm{cm}^{-1}\right)^{\mathrm{a}}$} & \multicolumn{2}{|c|}{$\Phi_{\text {fluo }}{ }^{\mathrm{a}}$} & \multicolumn{2}{|c|}{ Brightness $^{\mathrm{b}}$} & \multicolumn{2}{|c|}{$\mathrm{pK}_{\mathrm{a}}$} & \multirow{2}{*}{\multicolumn{2}{|c|}{$\begin{array}{l}\text { Actinic light } \\
(\mathrm{nm})\end{array}$}} & \multirow[t]{2}{*}{ Ref } \\
\hline \multicolumn{4}{|c|}{ PHOTOCONVERTIBLE FLUORESCENT PROTEINS (PCFPS) } & PRE & POST & PRE & POST & PRE & POST & PRE & POST & PRE & POST & & & \\
\hline mEos3.1 (M) & $\begin{array}{c}\begin{array}{c}\text { mEos2 } \\
{[\text { EosFP] }}\end{array} \\
\end{array}$ & L. hemprichii & HYG & $505 / 513$ & $570 / 580$ & 88.4 & 33.5 & 0.83 & 0.62 & $218 \%$ & $62 \%$ & 5.2 & 6.0 & \multicolumn{2}{|c|}{405} & [31] \\
\hline mEos3.2 (M) & $\begin{array}{c}\mathrm{mEos2} \\
{[\text { [EosFP] }}\end{array}$ & L.hemprichii & HYG & $507 / 516$ & $572 / 580$ & 63.4 & 32.2 & 0.84 & 0.55 & $159 \%$ & $53 \%$ & 5.4 & 5.8 & \multicolumn{2}{|c|}{405} & [31] \\
\hline mMaple (M) & mClavGR2 & Clavularia sp. & HYG & $489 / 505$ & $566 / 583$ & 15 & 30 & 0.74 & 0.56 & $33 \%$ & $50 \%$ & 8.2 & 7.3 & \multicolumn{2}{|c|}{405} & [41] \\
\hline pcDronpa (T) & Dronpa [22G] & $\begin{array}{c}\text { Echinophyllia } \\
\text { sp. SC22 }\end{array}$ & HYG & $505 / 517$ & $569 / 581$ & 115 & 75 & 0.85 & 0.68 & $291 \%$ & $190 \%$ & 5.5 & 6.3 & \multicolumn{2}{|c|}{405} & [15] \\
\hline pcDronpa2 $(\mathrm{T})$ & Dronpa [22G] & $\begin{array}{c}\text { Echinophyllia } \\
\text { sp. SC22 }\end{array}$ & HYG & $504 / 515$ & $569 / 583$ & 100 & 105 & 0.83 & 0.68 & $248 \%$ & $213 \%$ & 5.8 & 6.1 & \multicolumn{2}{|c|}{405} & [15] \\
\hline PSmOrange2 (M) & $\begin{array}{l}\text { mOrange } \\
\text { [DsRed] }\end{array}$ & Discosoma sp. & TYG & $546 / 561$ & $619 / 651$ & 51 & 18.9 & 0.61 & 0.38 & $95 \%$ & $23 \%$ & 6.6 & 5.4 & \multicolumn{2}{|c|}{488} & [22] \\
\hline \multicolumn{4}{|c|}{ REVERSIBLY SWITCHABLE FLUORESCENT PROTEINS (RSFPS) } & PRE & POST & PRE & POST & PRE & POST & PRE & POST & PRE & POST & on-off & off-on & \\
\hline$(-) \operatorname{modBFP}(\mathrm{M})$ & $\begin{array}{c}\text { mKalama1 } \\
{[\text { GFP] }}\end{array}$ & A. victoria & SYG & $390 / 455$ & NA & 27 & NA & 0.33 & NA & $26 \%$ & NA & 5.5 & ND & 405 & 514 & [29] \\
\hline$(-)$ mlrisGFP $(\mathrm{M})$ & IrisFP [EosFP] & L. hemprichii & HYG & $488 / 516$ & NA & 47 & 18 & 0.63 & NA & $89 \%$ & NA & 5.3 & $>10$ & 473 & 405 & [34] \\
\hline$(-)$ mGeos-M (M) & $\begin{array}{c}\text { mEos2 } \\
\text { [EosFP] }\end{array}$ & L. hemprichii & MYG & $503 / 514$ & NA & 51.6 & NA & 0.85 & NA & $131 \%$ & NA & $4.5-5.0$ & ND & 488 & 405 & [14] \\
\hline (-) rsEGFP2 (M) & EGFP [GFP] & A. victoria & AYG & $478 / 503$ & NA & 61 & NA & 0.30 & NA & $\sim 60 \%$ & NA & ND & ND & 488 & 405 & [28] \\
\hline (-) rsCherryRev $1.4(\mathrm{M})$ & $\begin{array}{c}\text { rsCherryRev } \\
\text { [DsRed] }\end{array}$ & Discosoma sp. & MYG & $572 / 609$ & NA & ND & NA & ND & NA & ND & NA & ND & 5.5 & 592 & 430 & [30] \\
\hline (+) PAiRFP1 (D) & $\begin{array}{c}\text { AtPCD } \\
\text { [AtBphP2] }\end{array}$ & $\begin{array}{l}\text { A. tumefaciens } \\
\text { C58 }\end{array}$ & billiverdin & $659 / 703$ & $690 / 717$ & 48.7 & 67.1 & NA & 0.048 & $9.6 \%$ & ND & 4.6 & 5.2 & - & $660 / 750$ & {$[40]$} \\
\hline (+) PAiRFP2 (D) & $\begin{array}{c}\text { AtPCD } \\
\text { [AtBphP2] }\end{array}$ & $\begin{array}{c}\text { A. tumefaciens } \\
\text { C58 }\end{array}$ & billiverdin & $657 / 708$ & $692 / 719$ & 39.5 & 63.6 & NA & 0.047 & $8.9 \%$ & ND & 4.7 & 5.4 & - & $660 / 750$ & [40] \\
\hline
\end{tabular}

Note: The table summarizes the photophysical properties of PTFPs prior (PRE) and after (POST) their phototransformation. It is noteworthy that in the last two years, to the authors' knowledge, no PAFPs have been developed.

a Abbreviations: $\lambda_{\max }$ ex/em, maximum of excitation/emission spectrum; $\varepsilon$, molar extinction coefficient; $\Phi_{\text {fluo }}$, fluorescence quantum yield.

${ }^{\mathrm{b}}$ Brightness is the product of fluorescence quantum yield and molar extinction coefficient expressed in \% of the EGFP brightness.

${ }^{c}$ wavelengths required for irreversible (PCFPs) or reversible (RSFPs) transitions.

$\mathrm{M}$, monomer; D, dimer; T, tetramer; NA, not applicable; ND, not determined. The sign of the photoswitching in RSFPs is depicted by (-) for negative and (+) for positive switching 
Click here to download high resolution image

(a)
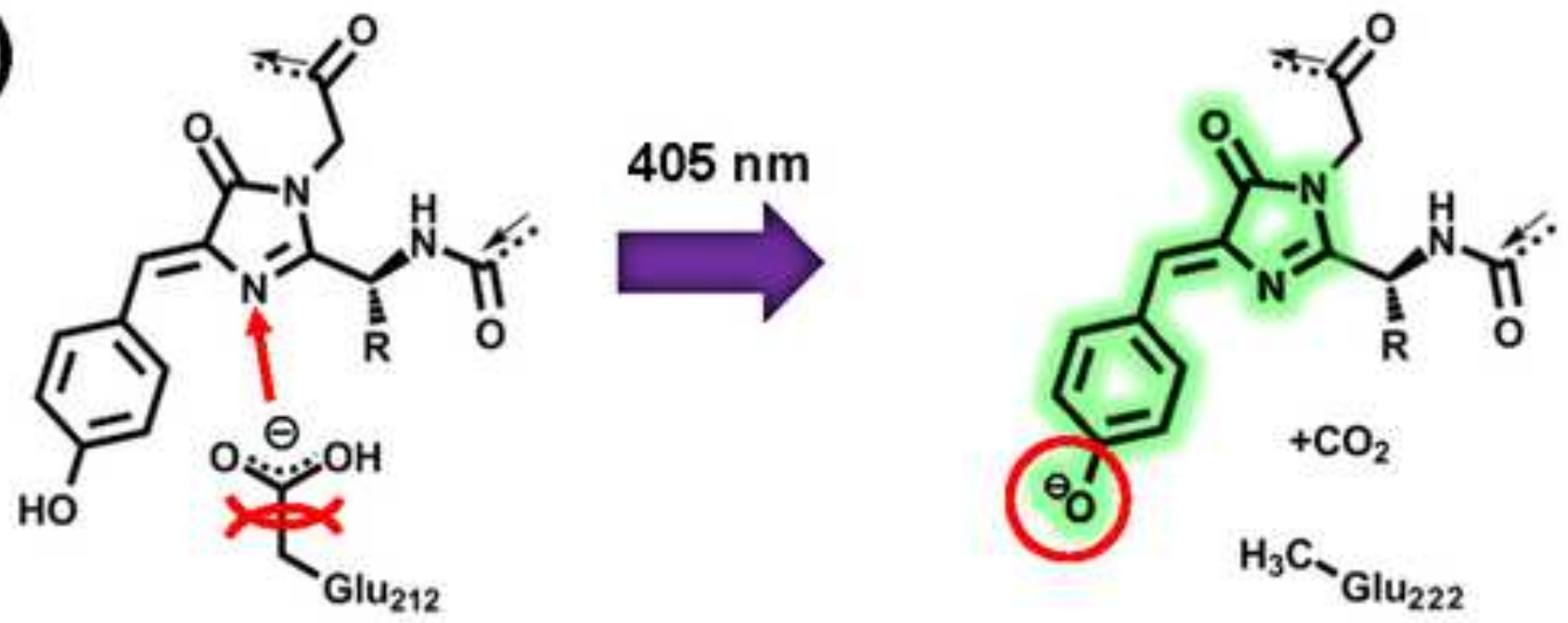

(b)
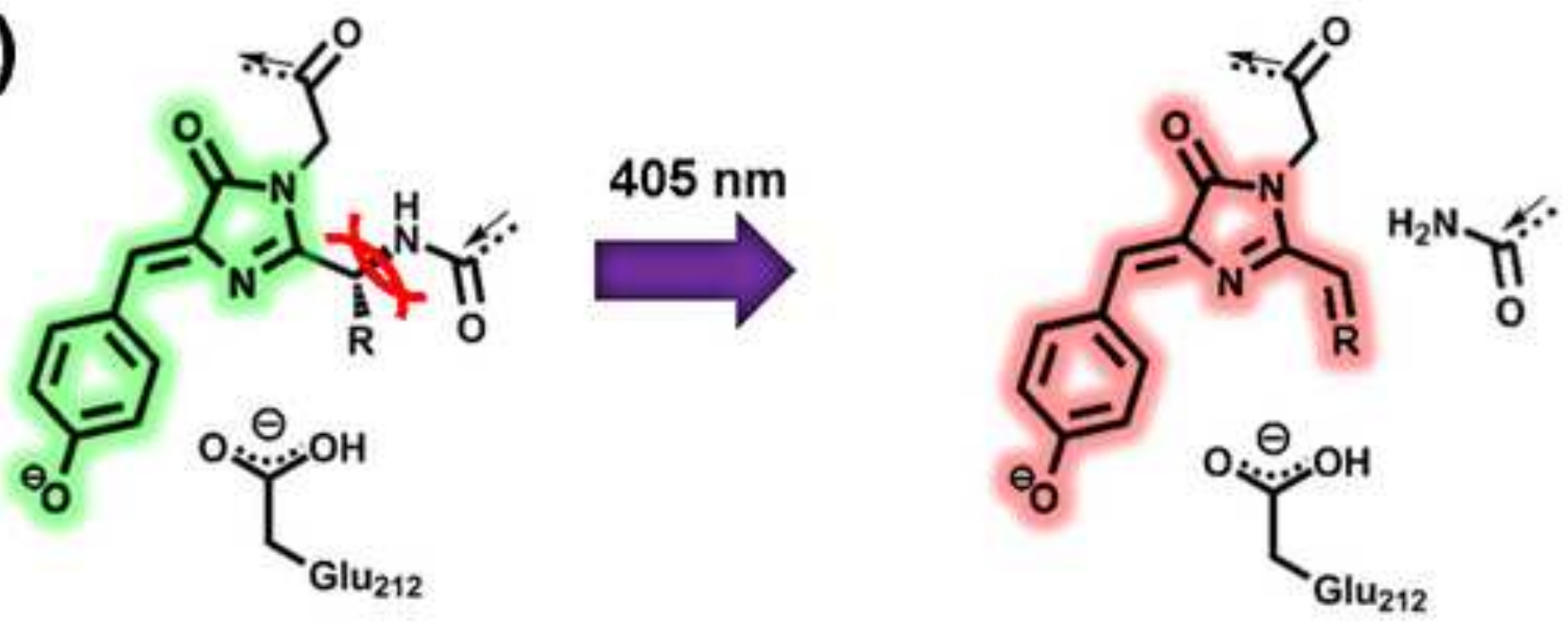

(c)
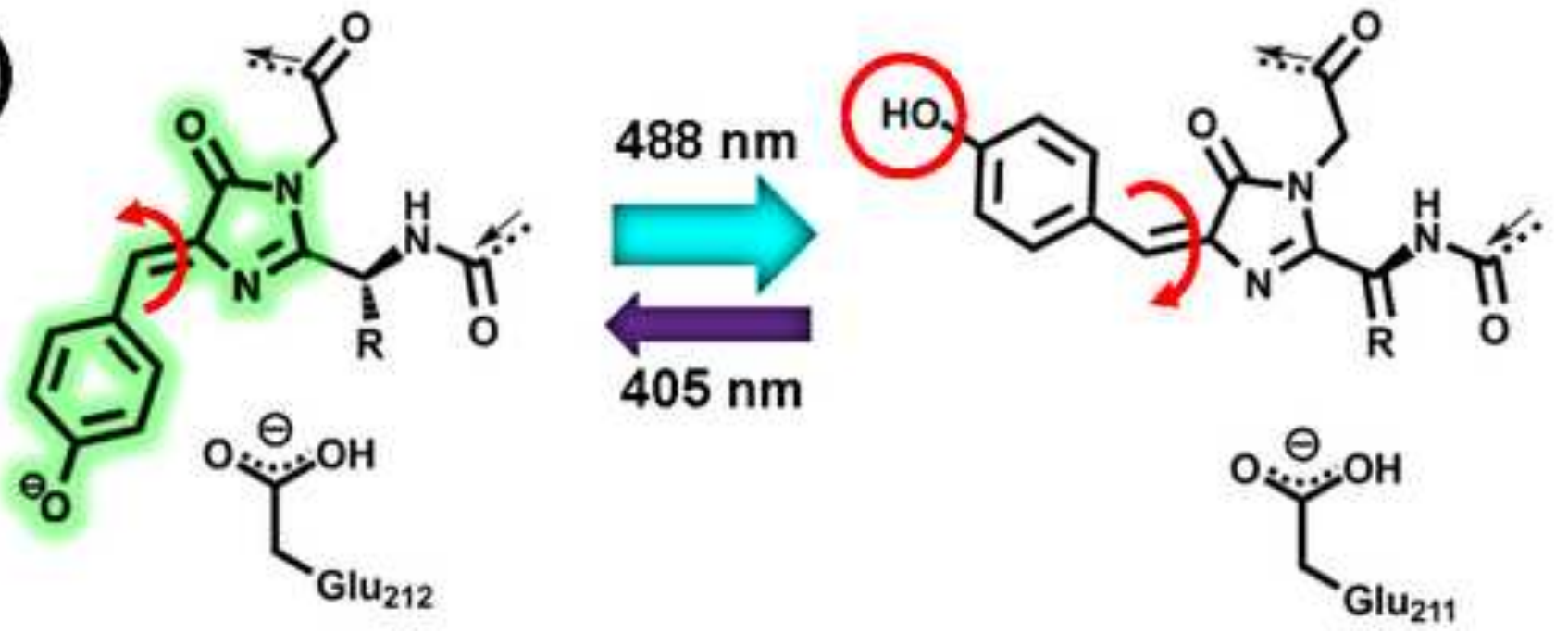

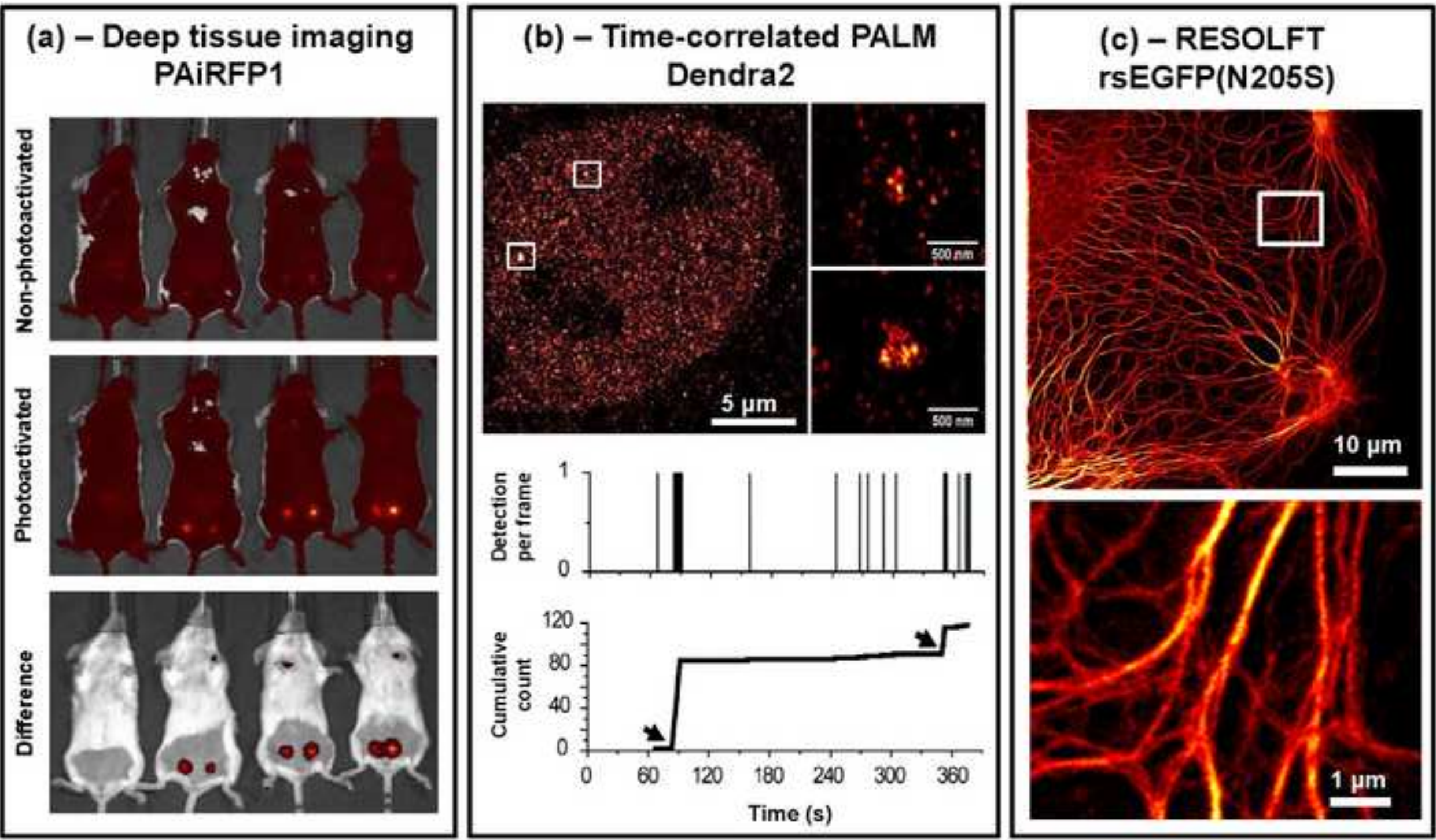
(a)
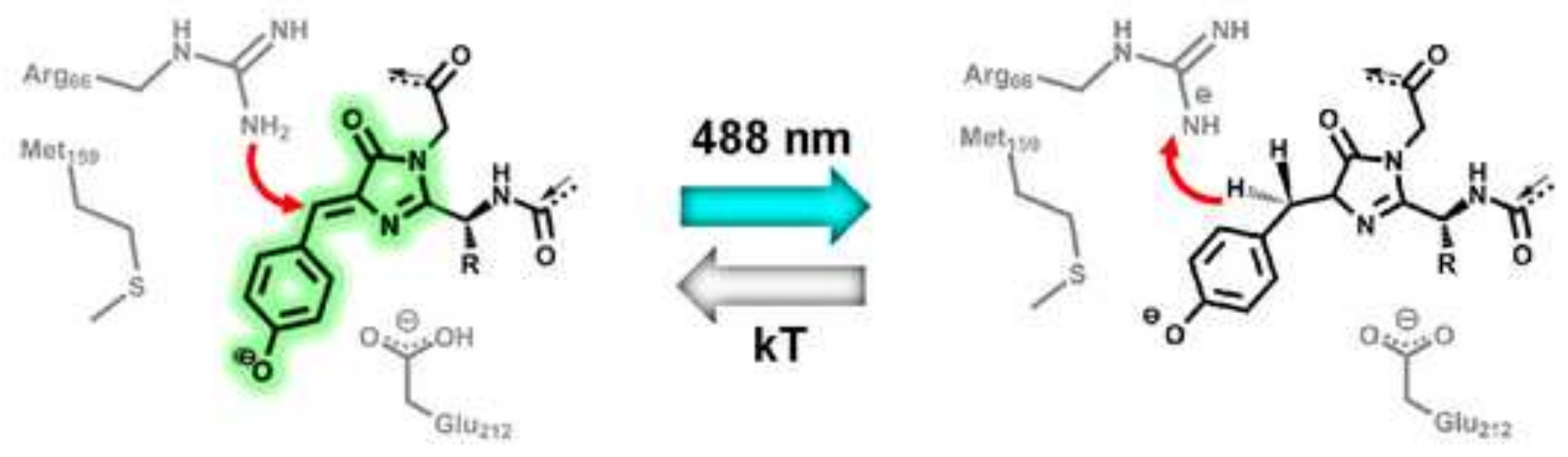

(b)
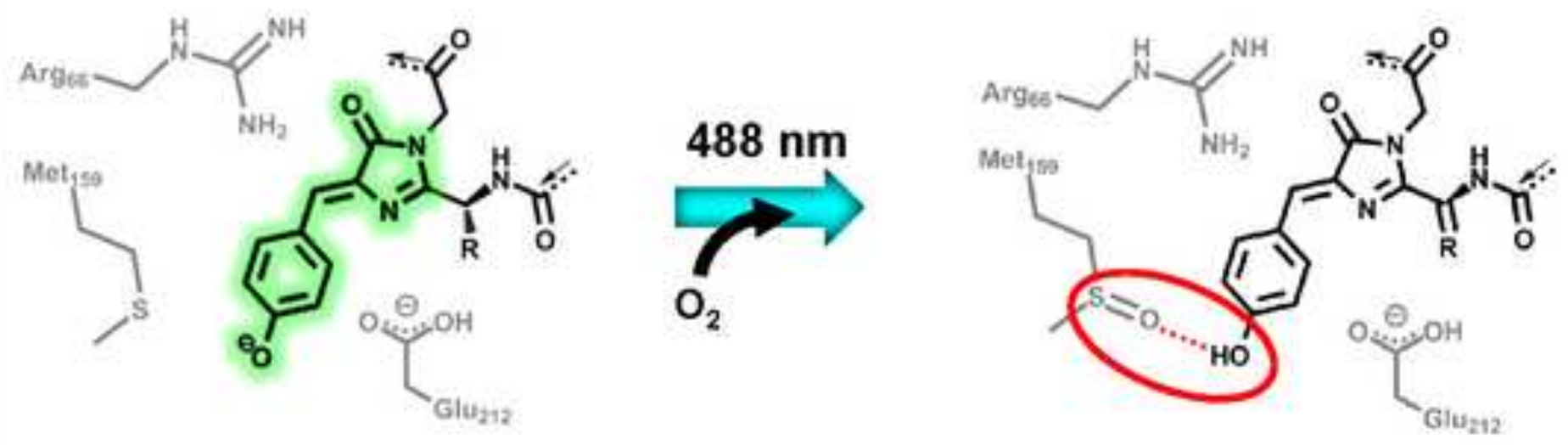

(c)
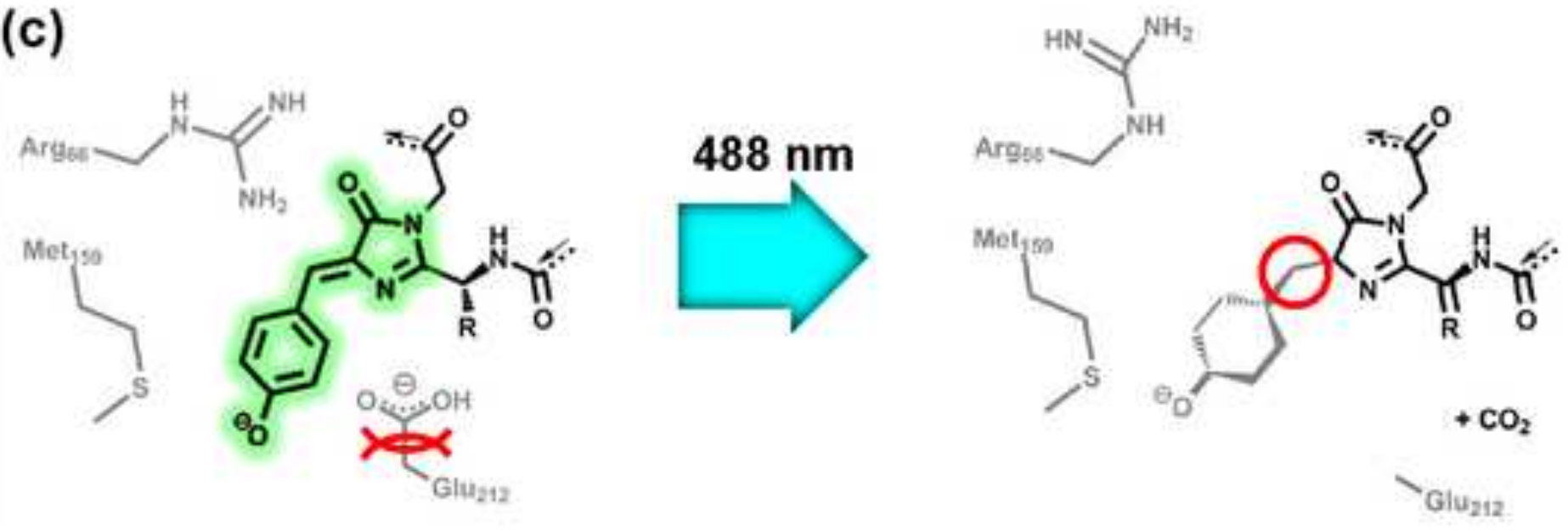
Click here to download high resolution image

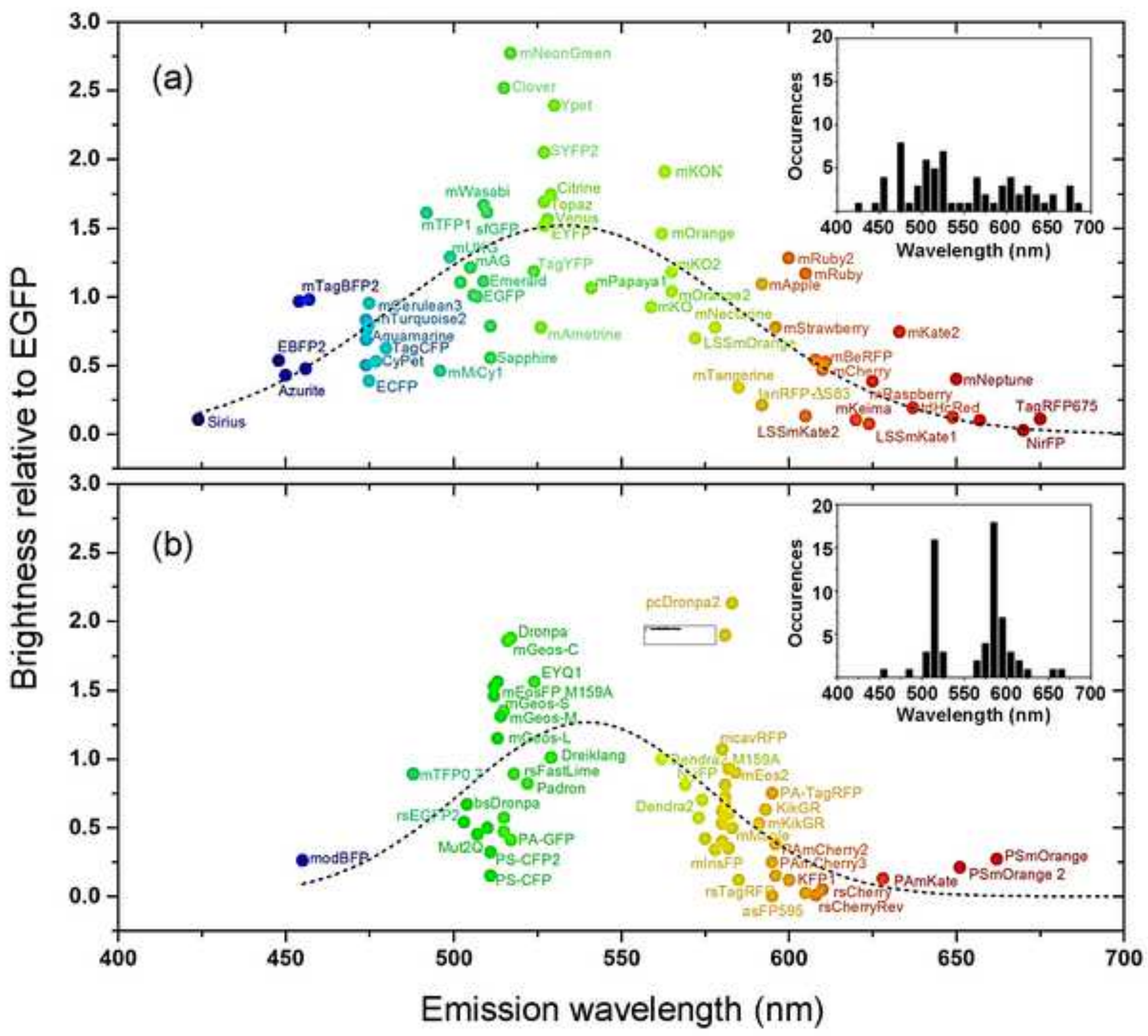

